\title{
Acknowledgement to Reviewers of Remote Sensing in 2016
}

\section{Remote Sensing Editorial Office}

Published: 12 January 2017

MDPI AG, St. Alban-Anlage 66, 4052 Basel, Switzerland; remotesensing@mdpi.com

The editors of Remote Sensing would like to express their sincere gratitude to the following reviewers for assessing manuscripts in 2016.

We greatly appreciate the contribution of expert reviewers, which is crucial to the journal's editorial process. We aim to recognize reviewer contributions through several mechanisms, of which the annual publication of reviewer names is one. Reviewers receive a voucher entitling them to a discount on their next MDPI publication and can download a certificate of recognition directly from our submission system. Additionally, reviewers can sign up to the service Publons (https://publons.com) to receive recognition. Of course, in these initiatives we are careful not to compromise reviewer confidentiality. Many reviewers see their work as a voluntary and often unseen part of their role as researchers. We are grateful to the time reviewers donate to our journals and the contribution they make.

If you are interested in becoming a reviewer for Remote Sensing, see the link at the bottom of the webpage http://www.mdpi.com/reviewers.

The following reviewed for Remote Sensing in 2016:

Aaboe, Signe

Aanstoos, James V.

Aaron, David

Aasen, Helge

Abadi, Shima

Abalos, Marta

Abarca-Hernandez, F.

Abbas, Sawaid

Abdel-Rahman, Elfatih

Abd-Elrahman, Amr

Abdul Aziz, Ammar

Abdullah, Meshal M.

Abe, Hiroto

Abrams, Michael J.

Achille, Cristiana

Adami, Marcos

Adler-Golden, Steven

Agapiou, Athos

Agarwal, Shefali

AghaKouchak, Amir
Ago, Expédit Evariste

Aguejdad, Rahim

Aguiar, Francisca

Aguilar, Manuel Ángel

Aguirre-Salado, Carlos A.

Ahl, Douglas E.

Ahmed, Bayes

Ahmed, Mohamed

Ahmed, Samir

Ahn, Yushin

Aiken, Rob

Aitken, Matthew L.

Akbari, Vahid

Al Kwatli, Mohamad Amer

Alam, Khan

Alavipanah, Sadroddin

Albano, Matteo

Alber, Merryl

Albergel, Clement

Alberti, Giovanni 
Albino, Fabien

Alcantara, Enner

Aleksandra, Sima

Aleksandrowicz, Sebastian

Alemohammad, Seyed Hamed

Alexander, Paul J.

Alexandre, Gonçalves

Alexandri, Georgia

Alexandridis, Thomas

Ali, Ahmed Loai

Ali, Behrangi

Ali, Iftikhar

Alim, Samat

Allan, Mat

Allasia, Paolo

Alleaume, Samuel

Almeida, Luis Pedro

Alonso, Luis

Alonso, María C.

Alonso- Montesinos, Joaquin

Alonso-Benito, Alfonso

Alonzo, Mike

Alpers, Werner

Alshawaf, Fadwa

Alvarez, Yuri

Álvarez, Orlando

Alvarez-Fernandez, Inmaculada

Aly, Mohamed

Al-Yaari, Amen

Amaral, Jorge

Amatulli, Giuseppe

Ambrosia, Vincent G.

Amditis, Angelos

Ames, Daniel

Amitrano, Donato

Amler, Esther

Amundarain, Aiert

An, Nan

Anagnostopoulou, Christina

Anderson, Christopher

Anderson, Ray G.

Anderson, Sharolyn

Andrade, Maria F.
Andrefouet, Serge

Andrews, Lauren

Aneece, Itiya

Angerer, Jay P.

Anghel, Andrei

Ansmann, Albert

Antonarakis, Alexander

Antropov, Oleg

Anyomi, Kenneth A.

Aplin, Paul

Arandjelović, Ognjen

Arcidiacono, Claudia

Ardila, Juan Pablo

Ardizzone, Francesca

Ardö, Jonas

Argüello, Francisco

Arii, Motofumi

Arkebauer, Timothy J.

Armenakis, Costas

Armenta, Sergio

Armitage, Richard

Armstrong, Edward

Armstrong, Roy

Arnó, Jaume

Aryal, Jagannath

Asam, Sarah

Ashouri, Hamed

Ashraf, Salman

Asilo, Sonia

Asner, Greg

Aspandiar, Mehrooz

Asrar, Ghassem

Athanassiadou, Maria

Atkinson, Nigel

Atzberger, Clement

Auer, Stefan

Augusto-Silva, Pétala B.

Aull, Brian F.

Avbelj, Janja

Avitabile, Valerio

Ayana, Essayas K.

A $\beta$ mann, Marc

Bachmann, Charles 


\begin{tabular}{|c|}
\hline Badawy, Moawad \\
\hline Bader, Markus \\
\hline Badgley, Grayson \\
\hline Baek, Stanley \\
\hline Bahl, Rajendar \\
\hline Bailey, David \\
\hline Baiocchi, Valerio \\
\hline Baird, Ian \\
\hline Baird, Mark \\
\hline Bajwa, Sreekala \\
\hline Baker, David \\
\hline Bakuła, Krzysztof \\
\hline Balali, Vahid \\
\hline Baldinelli, Giorgio \\
\hline Balenović, Ivan \\
\hline Ballesteros, Rocio \\
\hline Balletti, Caterina \\
\hline Balsas, Carlos \\
\hline Balz, Timo \\
\hline Balzarolo, Manuela \\
\hline Bandara, Ranmalee \\
\hline Bandeira, Lourenço \\
\hline Banerjee, Biplab \\
\hline Banerjee, Ruman \\
\hline Bankert, Richard L. \\
\hline Bao, Lifeng \\
\hline Bao, Shaowu \\
\hline Barbarella, Maurizio \\
\hline Barber-Meyer, Shannon \\
\hline Barbetta, Silvia \\
\hline Barbier, Nicolas \\
\hline Barbosa, Humberto A. \\
\hline Barbosa, Jomar Magalhães \\
\hline Bardi, Federica \\
\hline Barnes, Brian B. \\
\hline Barrado, Cristina \\
\hline Barron, John \\
\hline Barron, Olga \\
\hline Barsi, Julia A. \\
\hline Barthelmie, Rebecca \\
\hline Bartok, Blank \\
\hline Baselice, Fabio \\
\hline Bassa, Zaakirah \\
\hline
\end{tabular}

Bassani, Cristiana

Bastin, Jean-François

Bastin, Lucy

Basuki, T.M.

Battude, Marjorie

Baumann, Matthias

Bawazir, A. S.

Bayer, Anita

Bazi, Yakoub

Becek, Kazimierz

Beck, Marcus W.

Beck, Pieter

Beck, Richard

Becker, Rory

Beckers, Justin F

Bedini, Enton

Bégué, Agnés

Behera, Mukunda Dev

Behling, Robert

Behner, Florian

Beisl, Ulrich

Bekaert, David

Beland, Martin

Belgiu, Mariana

Bell, David

Bellvert, J.

Beltrán-Abaunza, Jose Maria

Benali, Akli

Bendig, Juliane

Bennett, Michael

Benoit, Alexandre

Berezowski, Tomasz

Berland, Adam

Bernabé, Sergio

Beşdok, Erkan

Bettinger, Pete

Bevan, Andrew

Bevly, David

Beyer, Hans-georg

Bhandari, Santosh

Bhateja, Vikrant

Bhatt, Rajendra

Bhattarai, Nishan 


\begin{tabular}{|c|c|}
\hline Bi, Jian & Bonin, Timothy \\
\hline Biagi, Ludovico & Bonnet, Stephanie \\
\hline Bian, Jinhu & Borak, Jordan S \\
\hline Bianchini, Silvia & Borfecchia, Flavio \\
\hline Bidel, Luc & Borsche, Michael \\
\hline Bienarz, Jakub & Boruff, Bryan J. \\
\hline Biggs, Trent & Boschetti, Mirco \\
\hline Biging, Gregory & Bosch-Lluis, Xavier \\
\hline Bignami, Christian & Böttcher, Kristin \\
\hline Bihl, Trevor J. & Bouali, Marouan \\
\hline Bilal, Muhammad & Bouchard, Michelle \\
\hline Bilbao, Julia & Boulet, G. \\
\hline Bilotta, Giuliana & Bourgeois, Jean \\
\hline Binder, Jann & Bovenga, Fabio \\
\hline Bisegna, Fabio & Bowles, Jeffrey H. \\
\hline Bishop, Michael & Bozzano, Francesca \\
\hline Bisquert, Mar & Bradley, Patrick Erik \\
\hline Bitelli, Gabriele & Brando, Vittorio \\
\hline Biudes, Marcelo & Brandt, Martin \\
\hline Błachowicz, Tomasz & Braun, Alexander \\
\hline Blackett, Matthew & Brazel, Anthony \\
\hline Blanc, Philippe & Brede, Benjamin \\
\hline Blankenship, Clay & Brehmer, Patrice \\
\hline Blaschke, Thomas & Breininger, David \\
\hline Błaszczak-Bąk, Wioleta & Bremer, Magnus \\
\hline Bleiweiss, Max & Brenning, Alexander \\
\hline Blesius, Leonhard & Bréon, François-Marie \\
\hline Blevins, Robert & Bresciani, Mariano \\
\hline Blonski, Slawomir & Breunig, Fábio Marcelo \\
\hline Bocchiola, Daniele & Brewer, Alan \\
\hline Boessen, Adam & Bright, Benjamin C \\
\hline Bohlin, Inka & Brilakis, Ioannis \\
\hline Bohlman, Stephanie & Brink, Andreas \\
\hline Böhm, Emanuele & Briottet, Xavier \\
\hline Boisvert, Linette & Brisco, Brian \\
\hline Bolch, Tobias & Brivio, Pietro Alessandro \\
\hline Bolovinou, A. & Brocca, Luca \\
\hline Bolten, Andreas & Brockmann, Carsten \\
\hline Bolvin, David T. & Brolly, Matthew \\
\hline Bonafoni, Stefania & Brooks, Evan B. \\
\hline Bonfante, Antonello & Brorsen, Wade \\
\hline Bonforte, Alessandro & Brotas, Vanda \\
\hline Boniface, Karen & Brovelli, Maria \\
\hline
\end{tabular}




\begin{tabular}{|c|c|}
\hline Brown, Molly & Cao, Fang \\
\hline Brucker, Ludovic & Cao, Yongfeng \\
\hline Brunn, Andreas & Capella, Juan Vicente \\
\hline Bruschini, Claudio & Capineri, Lorenzo \\
\hline Bryson, $\mathrm{M}$. & Capra, Alessandro \\
\hline Buccolieri, Riccardo & Carbajal Henken, Cintia \\
\hline Buchholz, Rebecca & Carbonneau, Patrice \\
\hline Buchroithner, Manfred & Carey, Drew A. \\
\hline Buckley, Joseph & Carlson, Tobby N. \\
\hline Buddenbaum, Henning & Carmona, Facundo \\
\hline Budge, Scott & Carmona, Pedro Latorre \\
\hline Budzynska, Maria & Carreño, M.F. \\
\hline Bue, Brian D. & Cartalis, Constantinos \\
\hline Bui, Dieu Tien & Carter, W.E. \\
\hline Buono, Andrea & Cartus, Oliver \\
\hline Burakowski, Elizabeth & Carvajal-Ramirez, Fernando \\
\hline Burgdorf, Martin & Carvalho, David \\
\hline Burns, John & Carvalho, Luis \\
\hline Buttafuoco, Gabriele & Case, Jonathan L. \\
\hline Byrd, Kristin & Caselles, Vicente \\
\hline Cachorro, Victoria E. & Castaldl, Fabio \\
\hline Cahalane, Conor & Castaldo, Raffaele \\
\hline Cai, Hongyan & Castañeda, Carmen \\
\hline Calamita, Giuseppe & Castellani, Beatrice \\
\hline Calcaterra, Domenico & Castellanos, Paola \\
\hline Calera Belmonte, Alfonso & Castellazzi, Giovanni \\
\hline Calle, Abel & Castle, Stephanie \\
\hline Calvão, Teresa & Cattani, Elsa \\
\hline Calvet, Jean-Christophe & Cavagnaro, Marta \\
\hline Camacho, Fernando & Cavalli, Rosa \\
\hline Cammarano, Davide & Cavanaugh, Kyle \\
\hline Campagnolo, Manuel & Cawkwell, Fiona \\
\hline Campana, Stefano & Cazenave, Anny \\
\hline Campbell, James B. & Ceddia, Marcos Bacis \\
\hline Campbell, James R. & Cen, Yi \\
\hline Campi, Cristina & Cerchio, Salvatore \\
\hline Campos, Isidro & Cerdá, Artemio \\
\hline Campos-Taberner, Manuel & Cerra, Daniele \\
\hline Candiani, Gabriele & Ch. Miliaresis, George \\
\hline Cánovas-García, Fulgencio & Chabrilat, Sabine \\
\hline Cansler, C. Alina & Chadwick, Bill \\
\hline Cantoro, Gianluca & Chakraborty, Bishwajit \\
\hline Cao, Changyong & Chan, Kam Wai Clifford \\
\hline
\end{tabular}


Chan, Pak Wai

Chan, Steven

Chand, Savin

Chang, Chung-Pai

Chang, Herng-hua

Chang, Jiyul

Chang, Kuo-Jen

Chang, Michael

Chang, Yi

Chang, Yuchun

Channan, Saurabh

Chanussot, Jocelyn

Chaplot, Vincent

Chapman, Daniel S.

Charou, Eleni

Chau, K.W.

Che, Huizheng

Cheema, M.J.M.

Chellasamy, Menaka

Chen, Alex

Chen, Chaur-Tzuhn

Chen, Chen

Chen, Chien-yuan

Chen, Fengrui

Chen, Jianyu

Chen, Jie

Chen, Jiquan

Chen, Jun

Chen, Mi

Chen, Nengcheng

Chen, Pengfei

Chen, Qi

Chen, Siwei

Chen, Xianfeng

Chen, Xuehong

Chen, Xuelong

Chen, Yushi

Chen, ZhiQiang

Cheng, Prof. Chi-Han

Cheng, Tao

Cheng, Weiming

CHENG, Yongcun

Chepushtanova, Sofya
Cherian, Ribu

Cherkauer, Keith

Cheruiyot, Elijah

Cheung, Jacob

Chevallier, Frédéric

Chiabrando, F.

Chiang, Jen-Shiun

Chiang, Jie-Lun

Chiang, Kai-Wei

Chini, Marco

Chiu, Long $\mathrm{S}$

Cho, Hyun Jung

Cho, Jaeil

Choi, Minha

Choi, Sungho

Choi, Yoonsuk

Choi, Yun Soo

Choi, Yunsoo

Cholewiak, Danielle

Chong, Geneva W.

Chow, Jacky

Chowdhury, Tanvir Ahmed

Christman, Zachary

Christodoulos, Chamzas

Chrysos, Michael

Chrysoulakis, Nektarios

Chu, Tianxing

Chuang, Ting-Wu

Chuang, Yung-Chung

Chudnovsky, Alexandra

Chum, Jaroslav

Churnside, James H.

Ciampalini, Andrea

Cigna, Francesca

Ciolli, Marco

Ciraolo, Giuseppe

Ciren, Pubu

Clarke, Keith

Clarke, Peter J.

Claverie, Martin

Clemente, Carmine

Clementini, Eliseo

Closson, Damien 
Cloutis, Edward

Coburn, Craig A.

Cocconcelli, Marco

Coddington, Odele

Cogley, Graham

Cogliati, Sergio

Colby, Jeffrey D.

Coll, César

Colliander, Andreas

Collin, Antoine

Collins, Michael

Colomina, Ismael

Comarazamy, Daniel

Comber, Lex

Combes, Didier

Comer, Douglas

Comín, Francisco A.

Comite, Davide

Cong, Xiaoying

Connette, Grant M.

Contreras-Medina, Luis

Convertino, Matteo

Conway, Susan

Conyers, Lawrence B.

Cook, Bruce

Cook, Monica

Coopersmith, Evan

Corner, Robert J.

Corona, Piermaria

Coscieme, Luca

Costa, J Miguel

Costa Surós, Montserrat

Costoya, $\mathrm{X}$.

Cotos, José M.

Cotten, David L.

Coudert, Benoit

Courty, Nicolas

Couteron, Pierre

Cozzolino, Daniel

Crago, Richard D.

Craig, Susanne

Crétaux, Jean-François

Crimmins, Theresa
Crisman, Thomas L.

Crosetto, Michele

Crosman, Erik T.

Cruise, James F.

Cudahy, Thomas

Cui, Shiyong

Cui, Song

Cunha, Mário

Cunliffe, Andy

Curci, Gabriele

Currenti, Gilda

Curtarelli, Marcelo

Da Silva, Bernardo B.

Da Silva, Jose

Dabboor, Mohammed

D'Addabbo, Annarita

Dai, Fei

Dai, Junhu

Dalponte, Michele

Damm, Alexander

Damodaran, Bharath Bhushan

Danaher, Tim

Dandois, Jonthan

Danescu, Radu Gabriel

Daniel, Jones

Danson, Mark

Darrozes, Jose

D'Asaro, Eric

Datta, Pawan

Davis, Curt

Davis, Frank

Dawod, Gomaa

De Bruin, Sytze

De Coning, Estelle

De Jong, Rogier

De Keersmaecker, Wanda

De Kloe, Jos

De Kok, Roeland

De Michele, Marcello

De Oliveira, Gabriel

De Paola, Francesco

De Ridder, Koen

De Teixeira Da Encarnação, João 
De Zavala, Miguel Angel

Dehecq, Amaury

Dehls, John

del Castillo, Edurne Martinez

Delalieux, Stephanie

Delashmit, Walter

Delegido, Jesús

Delgado, Jorge

Dell' Acqua, Fabio

Demaria, Eleonora

Demir, Begüm

Demirel, Cüneyd

Dempewolf, Jan

Denbina, Michael

Deng, Lei

Deng, Xiaoli

Deng, Yingbin

Denisov, Alexander

Deo, Ram Kumar

Der-Ming, Ma

Deseilligny, Marc Pierrot

Dessu, Shimelis B.

Detmers, R. G.

Deus, Dorothea

Devaney, John

Devanthery, Nuria

Devanthéry, Núria

Devi Kanniah, Kasturi

Devries, Ben

Dewan, Ashraf

Dharssi, Imtiaz

Dhungel, Ramesh

Di Martino, Gerardo

Di Paola, Francesco

Di Traglia, Federico

Diak, George R.

Dibdiakova, Janka

Dickson, Timothy

Didan, Kamel

Dierking, Wolfgang

Diesing, Markus

Dietz, Andreas J.

Dietze, Michael C.
Dimitriou, Elias

Dimitrov, Nikolay Krasimirov

Dimopoulou, Efi

Dimuccio, Luca Antonio

Diner, David

Ding, Yongjian

Diouf, Abdoul

Disney, Mathias

Dixon, Timothy

Dobreva, Iliyana

Doelling, David

Doherty, Owen

Dolan, Katelyn Ann

Dolder, Craig

D'Oleire-Oltmanns, Sebastian

Dong, Pinliang

Dong, Shaochun

Dong, Xiquan

Dong, Yusen

Doolan, Con

Dore, Nicole

Dou, Jie

Doulamis, Nikolaos D.

Dousset, Bénédicte

Doxani, Georgia

Doxaran, David

Dragozi, Eleni

Dragut, Lucian

Drăgut, Lucian

Drahor, Mahmut G.

Dronova, Iryna

Drummond, Mark A.

Du, Huaqiang

Du, Jinyang

$\mathrm{Du}$, Xiaomin

Duan, Hanchen

Duan, Hongtao

Dubovyk, Olena

Dungan, Jennifer L.

Durán-Alarcón, Claudio

Duro, Dennis C.

D'urso, Guido

Duveiller, Gregory 
Dworak, Volker

Dwyer, John

Dykema, John

Džubáková, Katarina

Eastin, Matthew D.

Ebmeier, Susanna K.

Eck, Thomas

Eckardt, Frank

Ediriweera, Sisira

Efremenko, Dmitry

Ehsan, Shoaib

Eineder, Michael

Eisenman, Ian

Ekonomou, Lambros

El Hajj, Mohamad

El Serafy, Ghada Y.

El-Alem, Anas

El-Askary, Hesham

Eldeiry, Ahmed

Elizabeth, Watson

El-Melegy, Moumen T.

Elmore, Andrew

Elsanabary, Mohamed Helmy

Elshall, Ahmed S.

Elsner, Paul

Eltner, Anette

Emelyanova, Irina V.

Emerson, Jay

Emil, Bayramov

Emmanuelle, Vaudour

Emmitt, George D

Eneva, Mariana

Erasmi, Stefan

Eresmaa, Reima

Eriksson, P.

Erives, Hector

Er-Raki, S.

Escolà, Alexandre

Escorihuela, María José

Espinoza-Molina, Daniela

Estornell, Javier

Evans, Ian S.

Everett, Mark
Fablet, Ronan

Fahnestock, Mark

Fairfield, Charlie

Faiz, Sami

Fakhri, Falah

Falcão-Flor, Ana Paula

Falcone, James A.

Fan, Chao

Fan, Fernando Mainardi

Fan, Hongchao

Fan, Jinlong

Fan, Ke

Fan, Xingwang

Fang, Bin

Fang, Chaoyang

Fang, Hongliang

Fang, Peng

Fanti, Riccardo

Fantini, Filippo

Faour, Ghaleb

Farr, Thomas

Farr, Tom

Farrand, William H

Farrell, Sinead L.

Fascetti, Fabio

Fassbinder, Joerg

Fassi, Francesco

Fassnacht, Fabian

Fassò, Alessandro

Fatih, Evrendilek

Fattahi, Heresh

Faugere, Yannice

Fayad, Ibrahim

Feber, Boris Le

Federica, Braga

Feigl, Kurt L.

Feist, Dietrich

Feldman, Daniel

Feliciano, Emanuelle

Feng, Lian

Feng, Liang

Feng, Min

Feng, Wanpeng 
Feng, Wei

Feng, Zhe

Fensham, Rod

Fensholt, Rasmus

Feret, Jean Baptiste

Fernandes, Richard

Fernandez, Roemi

Fernández, José Martínez

Fernandez-Manso, Alfonso

Ferraro, Angus J.

Ferraz, Antonio

Ferreira, Vagner G.

Ferrier, Graham

Ferro-Famil, Laurent

Fettweis, Michael

Fickas, Kate

Fieber, Karolina D.

Field, Donald

Filchev, Lachezar

Filippa, Gianluca

Filippetti, Ilaria

Fingas, Merv

Fiorentino, Costanza

Fiorucci, Federica

Fischer, Andrew

Fischer, Christoph

Fisher, Daniel K.

Fister, Iztok

Fleming, Kevin

Fletcher, Andrew

Fletcher, Reginald S.

Fletcher, Steven J.

Flores-de-Santiago, Francisco

Fokker, Peter A.

Fonseca, Ana

Foody, Giles

Ford, Phillip

Fore, Alexander

Forestier, Germain

Forkuor, Gerald

Forlani, Gianfranco

Forman, Barton

Forootan, Ehsan
Forouzanfar, Mohamad

Foucher, Samuel

Fourie, Christoffel

Fournier, Antoine

Foussekis, Dimitris

Foyo-Moreno, I.

Fraedrich, Klaus

Fraeman, Abigail A.

Francioni, M.

Frappart, Frédéric

Fratarcangeli, Francesca

Frattini, Paolo

Frazier, Amy E.

Frazier, Ryan

Freitag, Amy

Frelich, Lee

French, Andrew

Frey, Corinne

Frey, Julian

Fricker, Geoffrey A.

Friedt, Jean-Michel

Frontoni, Emanuele

$\mathrm{Fu}$, Dongjie

Fu, Peng

$\mathrm{Fu}$, Xiaolei

$\mathrm{Fu}$, Yuning

Fuell, Kevin

Fujibe, Fumiaki

Fujii, Yoshiaki

Fularz, Michal

Fumera, Giorgio

Furuya, Masato

Fusco, Giannetta

Fuse, Takashi

Gabellani, Simone

Gabellone, Francesco

Gabriel, Jose Luis

Gade, Martin

Gaetano, Raffaele

Gafurov, Abror

Gago, Xurxo

Gaiani, Marco

Gal, Tamas 
Galiatsatos, Nikolaos

Galidaki, Georgia

Gallaher, David

Gallay, Michal

Gallego, Javier

Gallego-Elvira, Belén

Galleguillos, Mauricio

Gallo, Kevin

Galvão, Lenio

Gama, Fábio Furlan

Gamba, Paolo

Gann, Daniel

Gao, Bocai

Gao, Feng

Gao, Huilin

Gao, Lianru

Gao, Xizhang

Gao, Yan

Garaba, Shungu

Garbarino, Matteo

Garcia, Pilar

Garcia-Gutierrez, Jorge

Garcia-Pedrero, Angel

Garcia-Santos, Vicente

Gardner, Chester S.

Garofalo, Pasquale

Garza, Rodrigo

Garzón, Gracia Ester Martín

Gastellu-Etchegorry, Jean-Philippe

Gatebe, Charles

Gaube, Peter

Gavrilov, Alexander

$\mathrm{Ge}$, Cui

Ge, Maorong

Ge, Yufeng

Gebbers, Robin

Geiger, Cathleen

Geipel, Jakob

Georgopoulos, Andreas

Georgoulias, Aristeidis

Gerard, France

Gerighausen, Heike

Gerke, Markus
Gerken, Tobias

Getzin, Stefan

Getzin, Stephan

Gevaert, Anouk

Gevaert, Caroline

Ghamisi, Pedram

Gharechelou, Saeid

Ghavidel, Ali

Gherboudj, Imen

Ghilain, Nicolas

Ghosh, Debarchana

Ghosh, S.K.

Ghosh, Tilottama

Ghuffar, Sajid

Ghulam, Abduwasit

Giampouras, Paris

Gianelle, Damiano

Giannaros, Theodore M.

Gibaldi, Agostino

Gibson, John

Gienko, Gennady

Gikas, Vassilis

Gilani, Hammad

Gillan, Jeff

Gillespie, Thomas

Gingrich, Simone

Ginzler, Christian

Giordan, Daniele

Giorgetti, Andrea

Gitelson, Anatoly

Giuliani, Roberta

Giulio Tonolo, Fabio

Gnyp, Martin L.

Goddijn-Murphy, Lonneke

Godone, Danilo

Gogu, Radu C.

Goïta, Kalifa

Gojda, Martin

Gokaraju, Balakrishna

Gökkaya, Kemal

Gokon, Hideomi

Gomez, Cristina

Gomez, Emilio 
Gómez, Luis

Gómez-Gutiérrez, Álvaro

Gómez-Rodríguez, Carola

Gommes, Rene

Gommes, René Alex

Gonçalves, Luisa M.S.

Gong, Adu

Gong, Boqing

Gong, Chongfeng

Gong, Jianhua

Gong, Wei

Gong, Wenlin

Gong, Wenyu

González, Higinio

González Dugo, Victoria

González Piqueras, José

González-Dugo, Patricia

Gonzalez-Mirelis, Genoveva

González-Rivero, Manuel

González-Roglich, Mariano

González-Sosa, Enrique

Gonzalez-Valdes, Borja

Gonzalo-Martín, Consuelo

Good, Elizabeth

Goodin, Douglas G.

Görgens, Eric B.

Gorte, Ben

Goshtasby, A. Ardeshir

Gosset, Marielle

Goulas, Yves

Gouveia, Celia

Gower, Jim

Gozalo, José Miguel Cuevas

Grammalidis, Nikos

Grandin, Raphael

Graser, Anita

Gravalos, Ioannis

Gray, Joshua

Graziano, Maria Daniela

Grecu, Mircea

Green, David R.

Green, Stuart

Grenzdörffer, Görres
Grima, Cyril

Grimaldi, Salvatore

Grinham, Alistair

Groen, Thomas

Grogan, Kenneth

Groom, Geoff

Gross, Barry

Grote, Anne

Grothe, Oliver

Grover, Aakriti

Gruber, Alexander

Gryning, Sven-Erik

Gryspeerdt, Edward

$\mathrm{Gu}$, Huan

Gu, Linjia

$\mathrm{Gu}$, Yanfeng

Gu, Zhu-Jun

Guan, Bin

Guan, Haiyan

Guarnieri, A. Monti

Guccione, Pietro

Guerra, Andre G. C.

Guerrero, Cesar

Guglielmino, Francesco

Guitet, Stéphane

Guo, Jian

Guo, Jinyun

Guo, Qinghua

Guo, Wanqin

Guo, Xulin

Guo, Zhongming

Gurshin, Christopher W.D.

Gusso, Anibal

Gutierrez, Benjamin T.

Gutiérrez-heredia, Luis

Gyllencreutz, Richard

Gyning, Sven-Erik

H.E. Epstein, H.E.

Haack, Barry

Haas, Eva Maria

Haberlie, Alex M.

Hackney, Chris

Hadjimitsis, Diofantos G. 


Haggerty, Julie
Hahn Chul, Jung
Halabisky, Meghan
Halfield, Jerry
Halios, Christos
Hall-Beyer, Mryka
Haller, Merrick
Hamm, Nicholas
Hammerling, Dorit
Hamunyela, Eliakim
Han, Hyangsun
Han, Junwei
Han, Youkyung
Hanan, Niall P.
Hancock, Steven
Hanes, Jonathan M.
Hanke, Klaus
Hänsch, Ronny
Hansen, Endre Hofstad
Hansen, Matthew
Hao, Wei
Hao, Xianjun
Harding, David
Hardy, Andrew
Harmel, Tristan
Harris, Ray
Haruyama, Shigeko
Harvey, E. Therese
Harwin, Steve
Hassan, Quazi
Hassan-Esfahani, Leila
Hata, Alberto
Hatten, James R.
Hauglin, Knut Marius
Havenith, Hans-Balder
Hayasaka, Hiroshi
Hayashi, Masato
Hayes, Daniel
Haywood, Andrew
He, Bin
He, Binbin
He, Chu
He, Fang
Hation

He, Fuhong

He, Jinxin

He, Liming

He, Mingzhu

He, Qisheng

He, Shaojun

$\mathrm{He}$, Tao

$\mathrm{He}$, Xianqiang

He, Yanyan

He, Yuhong

He, Zhi

Healey, Nathan C.

Healey, Sean P.

Heap, Michael J.

Hecobian, Arsineh

Hedley, John D.

Heege, Hermann J.

Heenkenda, Muditha K.

Heidemann, Karl

Heikkonen, Jukka

Hejazin, Yazan

Helble, Tyler A

Helm, Veit

Helman, David

Helmholz, Petra

Hemery, Lenaig G

Henderson, David

Henderson, Scott

Henebry, Geoffrey

Henry, Mary C.

Hermans, Thomas

Hernandez, Alexander

Hernandez, Monica

Hernandez-Marín, Martín

Hernández-Stefanoni, José Luis

Heron, Mal

Herrera, Gerardo

Herrera, Lorena P.

Herrmann, Jason

Herrmann, Stefanie

Hessner, Katrin

Hewison, Tim

Hewson, Michael 
Hewson, Robert

Heylen, Rob

Hieronymi, Martin

Higgins, Chris

Higuchi, Atsushi

Hilker, Thomas

Hill, Ross

Hillen, Florian

Hillger, Donald

Hilton, Timothy W

Hinterleitner, Alois

Hirata, Yasumasa

Hirose, Kiyoshi

Hissa, Leticia

Ho, Derrick

Ho, Hung Chak

Hoang, Kim Huong

Hobbs, Stephen

Hobi, Martina

Hodgetts, David

Hoelbling, Daniel

Hoffmeister, Dirk

Hofmann, Peter

Højstrup, Jørgen

Hole, Lars R.

Holifield Collins, Chandra D.

Hollaus, Markus

Hollmann, Rainer

Holt, Benjamin

Homayouni, Saeid

Homeyer, Cameron

Homolová, Lucie

Honda, Kiyoshi

Hong, Sang-hoon

Hong, Yang

Honkavaara, Eija

Hornbuckle, Brian

Horng, Shi-jinn

Hoshyaripour, Gholamali

Hosoda, Kohtaro

Hou, Xingsong

Howell, Steve

Howey, Meghan
Hsiao, Fu-yuen

Hsu, Feng Chi

$\mathrm{Hu}$, Baoxin

$\mathrm{Hu}$, Dewen

$\mathrm{Hu}$, Jun

$\mathrm{Hu}$, Jyr-Ching

$\mathrm{Hu}$, Qingwu

$\mathrm{Hu}$, tangao

$\mathrm{Hu}$, Xiuqing

$\mathrm{Hu}$, Xuefei

$\mathrm{Hu}$, Yingjie

Huang, Chang

Huang, Changchun

Huang, Chengquang

Huang, Guangwei

Huang, Guo H.

Huang, Jianping

Huang, Jianxi

Huang, Jingfeng

Huang, Lei

Huang, Mong-Han

Huang, Qingxu

Huang, Wenli

Huang, Xin

Huang, Yan

huang, yu

Hudak, Andrew

Huesca, Margarita

Huesca Martínez, Margarita

Huffman, George

Hufkens, Koen

Hughes, Topher

Hung, Ho-Lung

Hung, Ming-Chih

Hunsche, Mauricio

Hurni, Kaspar

Husak, Gregory J.

Husi, Letu

Hutjes, Ronald

Hütt, Christoph

Hwang, Paul A.

Hwang, Wen-Liang

Hyun, Chang-Uk 


\begin{tabular}{|c|c|}
\hline Iannacone, Jean Pascal & Je, Changsoo \\
\hline Iannini, Lorenzo & Jeganathan, C \\
\hline Ichii, Kazuhito & Jego, Guillaume \\
\hline Ichikawa, Kaoru & Jenerette, Darrel \\
\hline Ida, Maiello & Jeng, Yih \\
\hline Iervolino, Pasquale & Jeroen, Van Gent \\
\hline Iiames, John S. & Ji, Lei \\
\hline Iizuka, Kotaro & Jia, Kun \\
\hline Im, Jungho & Jiang, Chongya \\
\hline Imai, Nilton & Jiang, Dingde \\
\hline Imbrenda, Vito & Jiang, Dong \\
\hline Immitzer, Markus & Jiang, Liming \\
\hline Imperatore, Pasquale & Jiang, Lingmei \\
\hline Inamdar, Anand & Jiang, Weiguo \\
\hline Inglada, Jordi & Jiang, Xiaoguang \\
\hline Intrieri, Emanuele & Jiao, Weili \\
\hline Ioannidis, Charalabos & Jiapaer, Guli \\
\hline Iodice, Antonio & Jimenez-Munoz, Juan-Carlos \\
\hline Iordache, Marian-Daniel & Jin, Hongxiao \\
\hline Isada, Tomonori & Jin, Huiran \\
\hline Ishida, Haruma & Jin, $\mathrm{Li}$ \\
\hline Ishidaira, Hiroshi & Jin, Shuanggen \\
\hline Ishitsuka, Kazuya & Jin, Xiaomei \\
\hline Ismail, Riyad & Jo, Young-Heon \\
\hline Ito, Akihiko & Johansson, Therese \\
\hline Iungo, Giacomo Valerio & John, Ranjeet \\
\hline Ivanova, Natalia & Johnson, Brian Alan \\
\hline Iwao, Koki & Johnson, Lee F. \\
\hline Iwasaki, Akira & Joiner, Joanna \\
\hline Jaakkola, Anttoni & Jones, John \\
\hline Jacobsen, Karsten & Jones, Matt \\
\hline Jain, Meha & Jong, Gwo-Jia \\
\hline Jakubiec, J. Alstan & Jorge Ponzoni, Flávio \\
\hline Jamali, Sadegh & Jorgensen, Palle E.T. \\
\hline James, Michael & José, Marcato Junior \\
\hline Jamet, Cédric & Ju, Junchang \\
\hline Jandl, Robert & $\mathrm{Ju}$, Weimin \\
\hline Jánosi, Imre & Julitta, Tommaso \\
\hline Jaramillo, Fernando & Jun, Goo \\
\hline Jarihani, Ben & Jung, Andras \\
\hline Jarzabek-Rychard, Malgorzata & Jung, Hyung-Sup \\
\hline Jasek, Martin & Junttila, Virpi \\
\hline Jawak, Shridhar & Jupp, David \\
\hline
\end{tabular}




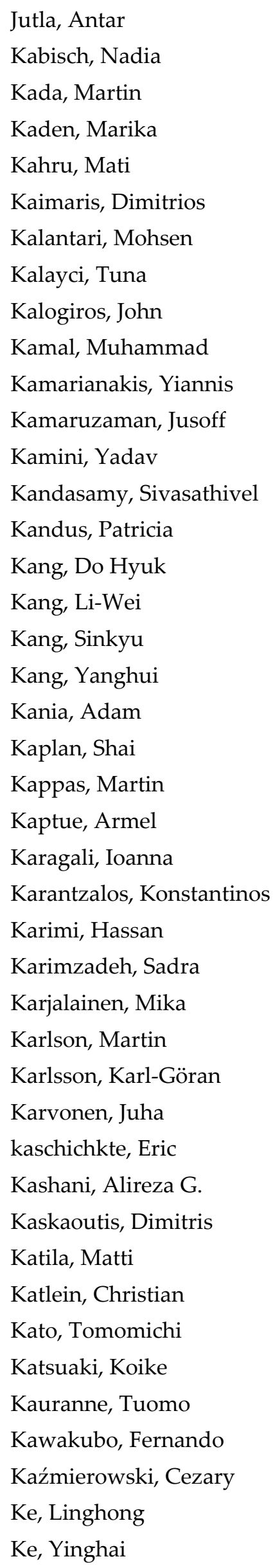

Kearney, Michael

Keckhut, Philippe

Kedzierski, Michal

Keegan, William

Kefauver, Shawn C.

Keith, Darryl J.

Kennedy, Robert

Keramitsoglou, Iphigenia

Kereszturi, Gabor

Kern, Stefan

Keßler, Carsten

Khalefa, Ehsan

Khan, Shuhab D.

Khanna, Shruti

Khatami, Reza

Khlopenkov, Konstantin

Khoshelham, Kourosh

Kiang, Richard

Kidwell, Autumn

Kiefer, Johannes

Kilpatrick, Kay

Kim, Do-Hyung

Kim, Jeong Woo

Kim, Jhoon

Kim, Jonghyuk

Kim, Jung-Rack

Kim, Keonwook

Kim, Sungwon

Kim, Sun-Hwa

Kim, Yeonjoo

Kim, Youngwook (UMT)

Kim, Youngwook (CSU-Fresno)

Kinch, Kjartan Münster

King, Bruce

King, Douglas

King, Joshua

Kinoshita, Tsuguki

Kiranoudis, Chris T.

Kirk, Ryan

Kirk-Davidoff, Daniel

Klein, Igor

Kleinebecker, Till

Klingbeil, Lasse 
Klisch, Anja

Knapp, Kenneth

Knudby, Anders

Knyazikhin, Yuri

Ko, Connie

Kobayashi, Hideki

Koch, Christian

Kociuba, Waldemar

Koedsin, Werapong

Koenig, Keith

Kogan, Felix

Kokalj, Žiga

Kolassa, Jana

Kolerski, Tomasz

Kolios, Stavros

Komatsu, Teruisha

Kondo, Masayuki

Kondo, Michelle

Kongoli, Cezar

Konings, Alexandra

Kontoes, Charalampos

Kopackova, Veronika

Kopačková, Veronika

Kopelevich, Oleg

Korets, Mikhail

Korpela, Ilkka

Kosasih, Buyung

Kosmopoulos, Dimitrios

Kosmopoulos, Panagiotis

Kostamovaara, Juha

Kosuke, Heki

Kothe, Steffen

Kouadio, Louis

Kourgialas, Nektarios

Kourkouli, Penelope

Koutsias, Nikolaos

Koutsias, Nikos

Koutsoudis, Anestis

Koyama, Yukinobu

Krakauer, Nir

Krishnamurthy, Raghavendra

Krishnan, Praveena

Krishnaswamy, Jagdish
Krofcheck, Dan

Kromer, Ryan A.

Kropacek, Jan

Krüger, Björn

Kruk, Michael

Krzhizhanovskaya, Valeria

$\mathrm{Ku}$, Nian-Wei

Kuai, Le

Kuang, Boyang

Kuang, Wenhui

Kubat, Ivana

Kudela, Raphael

Kuester, Michele

Kuffer, Monika

Kuhn, Nikolaus J.

Kulpa, Krzysztof

Kumar, Avishek

Kumar, Kanike

Kumar, Uttam

Kumar, Vipin

Kummerow, Christian D.

Kuntz, Steffen

Kurihara, Yukio

Kurum, Mehmet

Kussul, Nataliia

Kutser, Tiit

Kuze, Akihiko

Kvamme, Kenneth L.

Kwok, Ngai Ming

Kwon, Youngsang

Kyriakopoulos, Christodoulos

La, Hien Phu

Laakso, Kati

Laamrani, Ahmed

Lacava, Teodosio

Lacaze, Bernard

Ladefoged, Thegn

Laefer, Debra

Lagomasino, David

Lagüela, Susana

Lahoz, William A.

Lakshmi, Venkataraman

Lamb, Alistair 
Lambertini, Alessandro

Lampoltshammer, Thomas J.

Lamprecht, Sebastian

Landes, $\mathrm{T}$.

Landi, Tony C.

Landmann, Tobias

Landulfo, Eduardo

Langford, Zachary L.

Langhorne, Pat

Lanorte, Antonio

Lari, Zahra

Larkin, Andrew

Lasky, Ty

Lathrop Jr., Richard G.

Laurenza, Lucia Maria

Lavergne, Thomas

Laws, Edward

Lawston, Patricia

Lazecky, Milan

Lazzarini, Michele

Le, Chengfeng

Le Maire, Guerric

Le Mével, Hélène

Le Saux, Bertrand

Lechner, Alex

Ledo, Alicia

Lee, Eunmok

Lee, Hooyol

Lee, Hyongki

Lee, Jejung

Lee, Juheon

Lee, Jun-Hak

Lee, Saro

Lee, Seung Kuk

Lee, Yang-Won

Lefèvre, Sébastien

Lega, $\mathrm{M}$.

Leh, Mansoor D.

Lehmann, Jan Rudolf Karl

Lei, Lei

Lei, Ning

Lein, James

Leitinger, Georg
Leitner, Raimund

Lemmens, Mathias

Lemoine, Guido

Leng, Pei

Lensky, Itamar M.

Lentz, Erika

Leon, Javier

Leone, Antonio

Lepage, Richard

Lesiv, Myroslava

Leucci, Giovanni

Leverington, David W.

Levesque, Josee

Levin, Gregor

Levin, Noam

Levy, Robert

Lewiński, Stanisław

Leyk, Stefan

Lhermitte, Stef

Li, Aihua

Li, Cheng-Hsuan

Li, Deren

Li, Haiyan

Li, Jian

$\mathrm{Li}$, Jin

Li, Jun-Bao

$\mathrm{Li}, \mathrm{Li}$

$\mathrm{Li}$, Lin

Li, Peijun

Li, Qiangzi

Li, Rong

Li, Rui

Li, Sanmei

Li, Shao-kun

Li, Shihua

Li, Weidong

Li, Wenjuan

Li, Wenkai

Li, Xiaofeng

Li, Xiaoming

Li, Xiaoxiao

Li, Xinwu

Li, Xun 


\begin{tabular}{|c|c|}
\hline Li, Yangdong & Liou, Yuei-An \\
\hline Li, Yansheng & Lipoti, Jill \\
\hline Li, Yuan & Lipping, Tarmo \\
\hline Li, Yue & Litkey, Paula \\
\hline Li, Zengyuan & Liu, Guang \\
\hline Li, Zhao-Liang & Liu, Guosheng \\
\hline Li, Zhe & Liu, Hongyan \\
\hline Li, Zhe & Liu, James Jin-King \\
\hline Lian, Wenzhao & Liu, Kai \\
\hline Liang, Cunren & Liu, Kui \\
\hline Liang, Dong & Liu, Lin \\
\hline Liang, Fuyuan & Liu, Lingling \\
\hline Liang, Liang & Liu, Peng \\
\hline Liang, Lu & Liu, Qingsheng \\
\hline Liang, Xingming & Liu, Quanhua \\
\hline Liang, Xinlian & Liu, Shaomin \\
\hline Liao, Hung-chang & Liu, Sicong \\
\hline Liao, Wenzhi & Liu, Wei-Min \\
\hline Libohova, Zamir & Liu, Wen-Cheng \\
\hline Libonati, Renata & Liu, Xiaoming \\
\hline Liebisch, Frank & Liu, Xingzhao \\
\hline Lien, Ren-Chieh & Liu, Yaling \\
\hline Liesenberg, Veraldo & Liu, Yi \\
\hline Lieser, Jan & Liu, Yonggang \\
\hline Lilienthal, Holger & Liu, Zhaoyan \\
\hline Lillibridge, John & Liu, Zhihua \\
\hline Lim, Dong-Won & Liu, Zhong \\
\hline Lima, Fernando P. & Livens, Stefan \\
\hline Lin, Chin-Feng & Llorens Calveras, Jordi \\
\hline Lin, Feng-Cheng & Lo Conti, Francesco \\
\hline Lin, Wenming & Lohani, Bharat \\
\hline Lin, Xiangguo & Loisel, Hubert \\
\hline Lin, Yang & Loiselle, Steven A. \\
\hline Lin, Yucong & Lokers, Rob \\
\hline Lindberg, Eva & Long, David G. \\
\hline Lindenbergh, Roderik & Long, Di \\
\hline Lindner, Gerald & Long, Joseph \\
\hline Lindsley, Richard & Long, Nathalie \\
\hline Ling, Feng & Longo, Sandro \\
\hline Ling, Pui-Yu & Longoni, Laura \\
\hline Lingua, Andrea & López, Julia \\
\hline Linguet, Laurent & Lopez-Sanchez, Juan M. \\
\hline Linow, Stefanie & Lorenz, Eckehard \\
\hline
\end{tabular}


Lorenzo Navarro, Javier

Lorenzoni, Laura

Lorite, Ignacio

Los, Sietse

Lotsari, Eliisa

Lottering, Romano

Lovas, Tamás

Lovell, Jenny

Lowe, Kelsey

Lowell, Kim

Lu, Dan

Lu, Dengsheng

Lu, Hui

$\mathrm{Lu}$, Linlin

Lu, Yuhao

Luis Alvarez-Perez, Jose

Lukeš, Petr

Lund, Björn

Lundquist, Jessica

Luo, Pingping

Luo, Yi

Lyons, Evan A.

Lyons, Nathan

Dewan, Ashraf M.

Ma, Chunfeng

Ma, Lei

Ma, Lingling

Ma, Mingguo

Ma, Ronghua

Ma, Ting

Ma, Wei-chun

Ma, Weiqiang

Ma, Xuanlong

Ma, Yaoming

Maack, Joachim

Maaß, Nina

Macander, Matt

Macaulay, Gavin

MacDonald, Elizabeth

Macfadyen, Sarina

Madden, Marguerite

Madhavan, Bomidi Lakshmi

Maggioni, Viviana
Maghsoudi, Yasser

Magney, Troy

Magnusson, Martin

Mahecha, Miguel

Mahoney, Craig

Maignan, Fabienne

Maillard, Philippe

Maina, Joseph

Maingi, John

Makarau, Aliaksei

Maki, David

Malakar, Nabin K.

Malbeteau, Yoann

Malek, Žiga

Malenovsky, Zbynek

Malinka, Aleksey V.

Malinverni, Eva Savina

Mallinis, Giorgos

Mallorqui, Jordi J.

Malone, Brendan P.

Malone, Sparkle

Man, Cosmin D.

Mancini, Francesco

Manconi, Andrea

Mandal, Sohom

Mandanici, Emanuele

Mandlburger, Gottfried

Manevski, Kiril

Manfreda, Salvatore

Manfron, Giacinto

Mansberger, Reinfried

Mantas, Vasco.M.

Mantovani, Matteo

Manuela Portela, Maria

Manzo, Ciro

Mao, Rui

Mao, Zhihua

Marcello, Javier

Marchesini, Victoria A.

Marcotegui, Beatriz

Marghany, Maged

Mariani, Stefano

Marino, Armando 


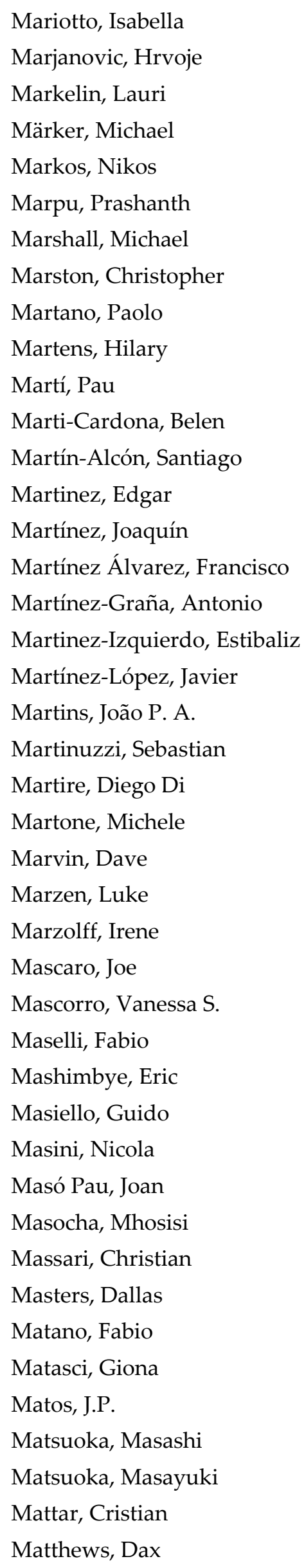

Matthews, Jessica

Maune, David

Mauro, Giovanni

Maxwell, Aaron

May, Stephane

Mayes, Sean

Maynard, Jonathan

Mazet, Vincent

Mberego, Seth

McCarthy, Matthew

McCarty, Jessica

McDougall, Kevin

McGovern, Mark

McKerrow, Alexa

McKinna, Lachlan

McNally, Amy

McNally, Kevin

McPherson, Meredith

McRoberts, Ronald

Mechri, Rihab

Meggio, Franco

Mehta, Manu

Mei, Yiwen

Meissner, Thomas

Mekhalfi, Mohamed Lamine

Melack, John

Melani, Samantha

Melendez-Pastor, Ignacio

Melo-Pinto, Pedro

Méndez, Valeriano

Mendiguren, Gorka

Menezes, Viviane V.

Meng, Jihua

Meng, Jinghui

Meng, Ran

Meng, Yu

Menna, Fabio

Meo, Pasquale De

Merényi, Erzsébet

Merete, Badger

Merino, Silvia

Mermoz, Stéphane

Merodo, José Antonio Fernandez 
Meroni, Michele

Mersey, Janet

Mertikas, Stelios

Mesas-Carrascosa, Francisco-Javier

Meschino, Simone

Metternicht, Graciela

Meyer, Kerry

Mialon, Arnaud

Michaelsen, Joel

Michishita, Ryo

Micijevic, Esad

Middleton, Elizabeth M.

Mielke, Christian

Mikada, Hitoshi

Mikhailova, Elena

Mikhelson, Ilya V.

Milenkovic, Milutin

Miles, Travis

Milewski, Adam

Milillo, Pietro

Militino, Ana Fernández

Millard, Koreen

Miller, Bradley A.

Miller, David W.

Mills, Steve

Mills, Steven

Minet, Christian

Ming, Hao

Miranda, Fernando

Miranda, Marcelo

Mirek, Katarzyna

Mirzaei, Parham

Mishra, Vikalp

Misra, Gourav

Mitchell, Catherine

Mitchell, Neil

Mitchell, Ross

Mitraka, Zina

Mitsopoulos, Ioannis

Mittaz, Jonathan

Miura, Hiroyuki

Miyazaki, Hiroyuki

Mlekuz, Dimitrij
Mo, Xingguo

Möckel, Thomas

Modica, Giuseppe

Moeller, Chris

Moghadas, Davood

Mogo, Sandra

Mohapatra, Rama Prasada

Moisan, Tiffany

Molin, Jose Paulo

Molina, Iñigo

Molina Gonzalez, Jose Luis

Molinario, Giuseppe

Møller-Jensen, Lasse

Moltchanova, Elena

Momm, Henrique

Mongus, Domen

Monserrat, Oriol

Monsivais-Huertero, Alejandro

Montagnani, Leonardo

Montanaro, Matthew

Montes, Marcos

Monti Guarnieri, Andrea Virgilio

Montorio, Raquel

Montoro Rodríguez, Amelia

Montpetit, Benoit

Moon, Todd

Moon, Twila A

Mooney, Peter

Moore, Angelyn W.

Moore, Nathan

Moore, Timothy S.

Moorthy, Inian

Mora, Carla

Morabito, Francesco Carlo

Morales, Luis

Morandeira, Natalia Soledad

Moreno, Max

Moreno, Miguel Angel

Moretti, Sandro

Morgan, Cristine

Moritsuka, Naoki

Morris, Edward P.

Morris, Tim 


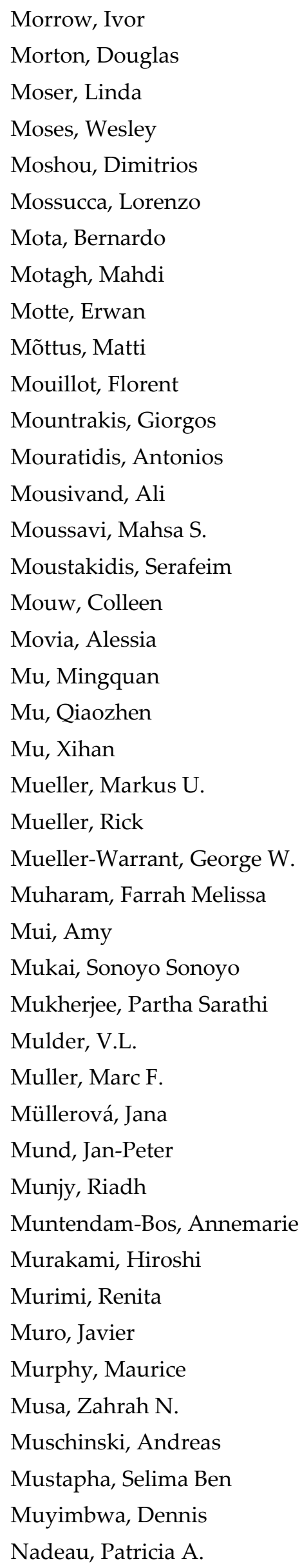

Naesset, Erik

Nagai, Shin

Nagarajan, Sudhagar

Nagol, Jyoteshwar R.

Nakaji, Tatsuro

Nakano, TomoKo

Nakhjiri, Navid

Nanni, Loris

Nanni, Marcos Raffael

Nardin, William

Nascetti, Andrea

Nassar, Ray

Natale, Antonio

Natraj, Vijay

Navarro, Ana

Navarro Fajardo, Juan Carlos

Navarro-Pedreño, Jose

Nazeer, Majid

Needoba, Joseph

Neeti, Neeti

Nehorai, Arye

Nekrasov, Alexey

Nelkin, Eric J.

Nerry, Françoise

Neumann, Mathias

Neumann, Patrick P.

Newlands, Nathaniel K.

Newman, Jennifer

Newman, Stuart M.

Newsam, Shawn

Newsom, Rob

Nex, Francesco

Neyland, Mark G.

$\mathrm{Ng}$, Alex Hay-Man

$\mathrm{Ng}$, Tuck Wah

Nguyen, Duy Ba

Nguyen, Phu

Nico, Giovanni

Nicolas, Le Corvec

Nield, Jo

Nielsen, Jonas Østergaard

Nielsen, Karina

Niemann, Jeffrey 
Niemeier, Wolfgang

Niemi, Milla

Ning, Xiaolin

Ningthoujam, Ramesh

Niro, Fabrizio

Nissi, Eugenia

Niu, Xutong

Nocerino, Erica

Noland, T.

Noland, Thomas L.

Nolè, Gabriele

Nord, Michael

Nordgren, Bryce

Norini, Gianluca

Norman, Steve

Notesco, Gila

Notti, Davide

Novelli, Antonio

Novellino, Alessandro

Novo, Evlyn

Nüchter, Andreas

Nuimura, Takayuki

Nyman, Petter

Nyström, Mattias

O'Connor, Brian

Obata, Kenta

Obryk, Maciej

O'Carroll, Anne G.

O'Connell, Jerome

O'Connell, Jessica L.

Oerke, Erich-Christian

Ogilvie, Andrew

Ogutu, Booker

Ohsawa, Teruo

Okarma, Krzysztof

Okujeni, Akpona

Okuyama, Arata

Okyay, Unal

Olaizola, Igor García

Oldeland, Jens

Olesen, Folke

Oliva, Diego

Oliva, Patricia
Oliveira, Julio Cesar

Olofson, Frans

Olofsson, Kenneth

O'Loughlin, Fiachra

Olsen, Michael J.

Olson, Lucretia E.

Olthof, Ian

Omrani, Hichem

O'neil-Dunne, Jarlath

Oneto, Luca

Ono, Keisuke

Onojeghuo, Alex Okiemute

Oommen, Thomas

Opitz, Rachel

Opoku-Duah, Stephen

Oppelt, Natascha

Orengo, Hector A.

Ørka, Hans Ole

Ortega Huerta, Miguel A.

Ortiz, Joseph D.

Osborn, Jon

Osés Eraso, Nuria

Ostendorf, Bertram

Otis, Daniel

Otkin, Jason A.

Ottle, Catherine

Ouchi, Kazuo

Oude Elberink, Sander

Ouled Sghaier, Moslem

Ouyang, Zutao

Ozdemir, Ibrahim

Oıkonomou, Emmanuel

Pacheco-Labrador, Javier

Pacheco-Martínez, Jesús

Pailhas, Yan

Pal, Mahesh

Pal, Sandip

Palafox, Leon

Paloscia, Simonetta

Palubinskas, Gintautas

Pampaloni, Paolo

Pan, Feifei

Pan, Zhigang 
Panagiotis, Elias

Panagopoulos, Thomas

Panda, Santosh K.

Panday, Prajjwal Kumar

Pantazi, Xanthoula Eirini

Papa, Maria Nicolina

Papakonstantinou, Apostolos

Papanikolaou, Ioannis

Papoutsis, Ioannis

Parajka, Juraj

Parcharidis, Isaak

Pardini, Matteo

Parece, Tammy E.

Pareeth, Sajid

Parente, Claudio

Parida, Bikash

Paris, Claudia

Paris, Paul J.

Parise, Mario

Parisi, Alfio

Park, Edward

Park, Sang-Eun

Park, Taejin

Parkinson, William

Parlow, Eberhard

Parmehr, Ebadat G.

Parrella, Giuseppe

Parrente, Mario

Parrish, Christopher

Pascual Castaño, Cristina

Pascucci, Simone

Pasolli, Edoardo

Pasquali, Paolo

Pateraki, Maria

Patias, Petros

Patzold, Stefan

Paudel, Uttam

Paul, George

Paulo Jose, Murillo-Sandoval

Pavlidis, Ioannis

Pecci, Antonio

Pedrero, Angel García

Peng, Ge
Peng, Jian

Peng, $X i$

Peng, Yi

Pengra, Bruce $W$.

Penna, N. T.

Penner, Margaret

Pepe, Antonio

Pepe, Monica

Pepe, S.

Percival, Jeanne

Perera, Kithsiri

Perez, Francesca

Pérez Cruzado, César

Pérez González, Pérez González

Perez-Ramirez, Daniel

Pergola, Nicola

Perko, Roland

Perry, Charles

Persello, Claudio

Persico, Raffaele

Persson, Henrik

Petcu, Dana

Peteinatos, Gerassimos

Peterson, Seth H.

Peterson, Urmas

Petrakis, Roy E.

Petropoulos, George

Petroselli, Andrea

Pettita, Marcello

Petty, Alek

Pfeffer, Julia

Picco, Lorenzo

Pickell, Paul

Pickens, Bradley A.

Pickering, Mark

Picotte, Joshua J.

Pieper, Michael

Pieraccini, Massimiliano

Pierce, Leland E.

Pierce Jr., Kenneth B.

Pieri, David

Piermattei, Livia

Pietrafesa, Len 
Pimont, François

Pina, Pedro

Pingel, Thomas J.

Pinnel, Nicole

Pinotti, Lucio P.

Pinto, Naiara

Pinzón, Jorge

Pio, Gianvito

Piras, Andrea

Pisano, Andrea

Pisek, Jan

Piskozub, Jacek

Pitkänen, Timo

Pizarro, Marta Béjar

Pizzo, Silvio Del

Plank, Simon

Plant, Richard E.

Plutzar, Christoph

Pôças, Isabel

Podobnikar, Tomaž

Poggi, Giovanni

Pohjola, Vejio

Pohl, Christine

Poland, Mike

Pollino, Maurizio

Polo, Jesus

Pölönen, Ilkka

Ponzoni, Flavio

Pope, Allen

Poppenga, Sandra K.

Porcu', Federico

Posluschny, Axel G.

Potapov, Peter

Pottier, Julien

Pouliot, Darren

Pourghasemi, Hamid Reza

Poursanidis, Dimitris

Powell, Rebecca

Powell, Scott

Prasad, Narasimha S.

Prasath, V. B. Surya

Prata, A. J.

Pratihast, Arun
Pratola, Chiara

Prats, Pau

Prestininzi, P.

Prezioso, Giuseppina

Price, Bronwyn

Price, Owen F.

Pricope, Narcisa

Priem, Frederik

Prigent, Catherine

Proctor, Chris

Przybilla, Heinz-Jürgen

$\mathrm{Pu}$, Ruiliang

Pudelko, Rafal

Puigdefabregas, Juan

Puletti, Nicola

Pullanagari, Reddy R.

Purves, Ross

Puschell, Jeff

Pytharoulis, Ioannis

Qi, Feng

Qi, Yi

Qi, Youcun

Qi, Zhixin

Qiao, Zhijun

Qin, Cheng-Zhi

Qin, Rongjun

Qin, $Y i$

Qin, Yuanwei

Qin, Zhi-Hao

Qingli, Luo

Qiu, Jianbin

Qiu, Jiangxiao

$\mathrm{Qu}$, Ying

$\mathrm{Qu}$, Yonghua

Qu, Zhipeng

Quan, Jinling

Quan, Xingwen

Quental, Lidia

Quinn, Nigel W.T.

Racault, Marie-Fanny

Radoux, Julien

Rahimzadeh, Parinaz

Rahman, Ataur 


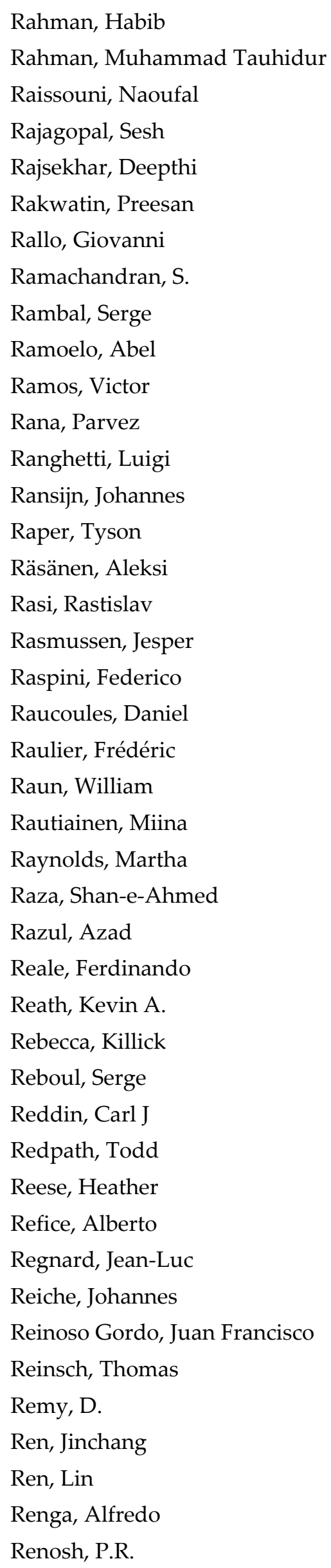

Renza, Diego

Resop, Jonathan

Retalis, Adrianos

Reulke, Ralf

Revuelto, Jesús

Reyes, Melissa

Rezaee, Mohammad

Rial, Fernando

Riaza, Asuncion

Riccioli, Francesco

Richard, Cedric

Richard, Crout

Riche, Andrew B.

Richter, Rudolf

Ricker, Robert

Riédi, Jérôme

Rieg, Lorenzo

Riffler, Michael

Rigge, Matthew

Riggs, George

Riley, Karin L.

Ringerud, Sarah

Rinne, Eero

Riquelme, Adrián

Risbøl, Ole

Ritchie-Tyo, Liz

Ritter, Christoph

Rivard, Benoit

Rivas-Casado, Monica

Robert, Stavn

Rocca, Fabio

Röder, Achim

Rodger, James A.

Rodrigo, Javier Sanz

Rodrigues, Arlete

Rodriguez, Nemesio

Rodríguez, Dionisio

Rodríguez Gonzálvez, Pablo

Rodríguez-Martín, Manuel

Rodriguez-Moreno, Victor M.

Roelofsen, Hans

Roggero, Marco

Roig, Ignacio 
Rojas, Carlos

Rojas, Oscar

Rolfe, Stephen Alexander

Romo Leon, Jose Raul

Roncat, Andreas

Roncella, Riccardo

Rönnholm, Petri

Rontogiannis, Athanasios

Rooijen, Nils M. Van

Rosario, Dalton

Roscher, Ribana

Rosina, Elisabetta

Rossi, David

Rossow, Bill

Roth, Keely

Rott, Helmut

Roujean, Jean-louis

Roumet, Pierre

Roundy, Joshua K.

Rousseau, David

Rousseaux, Cecile S.

Rowell, Eric

Rowlandson, Tracy

Roy, Alexandre

Roy, David

Roy, Parth Sarathi

Rozenstein, Offer

Ruano, António

Rubio Caballero, Eva María

Rucci, Alessio

Rudemo, Mats

RuizArmenteros, Antonio Miguel

Running, Steve

Runping, Runping

Russo, Paolo

Rutgersson, Anna

Ryu, Donghyeon

Ryzhkov, Alexander

Saarela, Svetlana

Saarinen, Ninni

Saavedra, Mario Lillo

Sabol, Donald

Sadeghi, Morteza
Sadjadi, Firooz

Saeed, Yahyanejad

Saepuloh, Asep

Safacas, Athanasios

Sahoo, Alok K.

Saibi, Hakim

Sakuno, Yuji

Sakurada, Ken

Salama, Suhyb

Salamí, Esther

Salas, Eric Ariel L.

Salaza, Alvaro

Salazar, Addisson

Salehi, Bahram

Saleri, Renato

Salerno, Giuseppe

Salk, Carl

Salvatore, Mark

Salvia, Mercedes

Salvini, Riccardo

Samczynski, Piotr

Samiappan, Sathishkumar

Samimi, Cyrus

Sampietro, Daniele

Sanada, Yukihisa

Sanchez, Nilda

Sánchez De Miguel, Alejandro

Sanctis, Mauro De

Sander, Lino

Sandra, Jakob

Sandric, Dr. Ionut

Sankey, Temuulen

Sano, Edson

Santagati, Cettina

Santangelo, Michele

Santi, Emanuele

Santis, Alberto De

Santos, Jefersson A. Dos

Santos Teixeira, Adunias Dos

Santos-Assunção, Sónia

Sarkissian, Alain

Sarmiento, Alvaro Lau

Sarris, Apostolos 
Sathyendranath, Shubha

Satori, Shin

Saul, Torrico

Save, Himanshu

Savi, Patrizia

Savin, Igor

Sayer, Andrew Mark

Scalenghe, Riccardo

Scarpace, Frank L.

Schack-Kirchner, Helmer

Schaefer, Kevin

Schaefer, Klaus

Scharien, Randall K.

Schepaschenko, Dmitry

Schindler, Stefan

Schirrmann, Michael

Schlaepfer, Daniel

Schlund, Michael

Schmid, Thomas

Schmidt, Frederic

Schmitt, Andreas

Schmitt, François G.

Schmitt, Michael

Schmittgen, Simone

Schneemann, Jörge

Schneibel, Anne

Schneider, David J.

Schneider, Fabian D.

Schneider, Mathias

Schneider, Sven

Schröder, Dietrich

Schroeder, Dustin M.

Schull, Mitch

Schumacher, Johannes

Schumann, Guy

Schwartz, Craig

Schwarz, Gottfried

Schweiger, Axel

Schweitzer, Christian

Scott, Andrea

Scotti, Roberto

Scozzari, Andrea

Scudiero, Elia
Scussolini, Paolo

Sczypinski, Piotr

Sedano, Fernando

Seddon, Alistair

See, Linda

Seehaus, Thorsten

Seekell, David

Seen, Danny Lo

Segoni, Samuele

Seidel, Dominik

Selkowitz, David

Sellitto, Pasquale

Selva, Massimo

Selvarajan, Sowmya

Senf, Cornelius

Seoane, Lucia

Sequeira, Ana Martins

Serbin, Guy

Serio, Carmine

Serna, Andrés

Sessa, Salvatore

Seyler, Frédérique

Shafian, Sanaz

Shafri, Helmi

Shahabfar, Alireza

Shahtahmassebi, Amirreza

Shahzad, Muhammad

Shamir, Eylon

Shane, David Grahame

Shang, Kun

Shang, Ronghua

Shang, Songhao

Shanley, Lea

Shao, Changliang

Shao, Guofan

Shao, Hongbo

Shao, Quanqin

Shao, Xiaowei

Shao, Yang

Shao, Zhenfeng

Shapiro, Aurélie

Sharif, Hatim

Sharma, JB 
Sharma, Ram C.

Sharma, Vivek

Sheeren, David

Sheffield, Kathryn

Shelestov, Andrii

Shen, Fang

Shen, Huanfeng

Shen, Linlin

Shen, Suhung

Shen, Zhenxi

Shendryk, Iurii

Sherrard, Mark

Shi, Kun

Shi, Lei

Shi, Runhe

Shi, Wei

Shi, WenJiao

Shi, Xun

Shi, Zhenwei

Shih, Peter Tian-yuan

Shilling, Fraser

Shim, Changsub

Shinoda, Toshiaki

Shiyomi, Masae

Shkvarko, Yuriy

Shore, Jennifer A.

Short, Naomi

Shoshany, Maxim

Shrestha, Uttam Babu

Shum, CK

Shuvankar, Ghosh

Siachalou, Sofia

Sibanda, Mbulisi

Siderius, Christian

Sidiropoulos, Pantelis

Sieber, Anika

Sieglaff, Justin

Silva, Thiago Sanna Freire

Sim, Sunhui

Simeonakis, Elias

Simila, Markku

Simley, Eric

Simms, Daniel
Sims, Neil

Singh, Aditya

Singh, Kunwar K.

Singh, Ramesh K.

Singh, Randhir

Singh, Sudhir Kumar

Singh, Upendra

Sismanidis, Panagiotis

Sithole, George

Skakun, Sergii

Skierucha, Wojciech M.

Skinner, Christopher

Skourup, Henriette

Škrinár, Andrej

Slade, Wayne Homer

Slonecker, Terrence

Small, David

Smedt, Philippe De

Smith, G.1.

Smith, Kathryn

Snow, Nathan P

Soares, Amilcar

Sobiech-Wolf, Jennifer

Socha, Jaroslaw

Sodoudi, Sahar

Sofia, Giulia

Sogacheva, Larisa

Sohel, Ferdous

Sohn, Gunho

Sohn, Hong-Gyoo

Soja, Maciej Jerzy

Solaro, Giuseppe

Solberg, Svein

Soliman, Aiman

Solimene, Raffaele

Somers, Ben

Son, Nguyen-Thanh

Sona, Giovanna

Song, Chunqiao

Song, Danxia

Song, Shalei

Song, Xiang

Song, Xiao-Peng 
Sonnemann, Till Frieder

Sørensen, Louise Sandberg

Soroosh, Sorooshian

Sosik, Heidi

Sousa, Joaquim

Souza, Carlos

Souza, Paulo De

Spagnolo, Matteo

Spak, Scott

Spanhove, Toon

Spencer, Michael W.

Spiliotopoulos, Marios

Spinoni, Jonathan

Spivak, Arthur

Spizzichino, Valeria

Spreafico, Margherita Cecilia

Sreekanth, Vakacherla

Srinivas, Umamahesh

Srivastava, Prashant K.

Srivastava, Sanjeev

Stabile, Luca

Stacey, Peter

Ståhl, Göran

Stamenkovic, Jelena

Stanev, Emil

Stanier, Charles

Statella, Thiago

Stavrakoudis, Dimitris G.

Steele, Caiti

Stefano, Ferraris

Steffen, Holger

Stehman, Stephen

Steiniger, Stefan

Stellmes, Marion

Stengel, Martin

Stentoumis, Christos

Stephani, Henrike

Stephen, Haroon

Sterckx, Sindy

Stereńczak, Krzysztof

Steven, Michael

Stewart, Iain Douglas

Stiff, Doug
Still, Chris

Stoffelen, Ad

Stolz, Roswith

St-Onge, Benoît

Stopa, Justin E.

Stopforth, Riaan

Storch, Tobias

Storey, James

Stow, Doug

Strobl, Peter

Stumpf, Andre

Stumpf, Rick

Štych, Přemysl

$\mathrm{Su}$, Chun-Hsu

$\mathrm{Su}$, Lihong

$\mathrm{Su}$, Tung-Ching

$\mathrm{Su}, \mathrm{Xin}$

$\mathrm{Su}$, Yuan-Fong

Suarez, Alvaro

Suchenwirth, Leonhard

Suh, Myoung-Seok

Suī, Haigang

Sullivan, Franklin

Sun, Alexander

Sun, Danfeng

Sun, Jian

Sun, Ling

Sun, Qingsong

Sun, Ranhao

Sun, Shaohui

Sun, Weihua

Sun, Weiwei

Sutanto, Samuel Jonson

Suwa, Rempei

Svigkas, Nikos

Svoma, Bohumil M.

Swan, Chantal

Swartz, William

Syed, Abdul Haleem

Sykioti, Olga

Szabó, Szilárd

Szantoi, Zoltan

Szewczyk, Roman 
Szporak-Wasilewska, Sylwia

Szulwic, Jakub

Szypłowska, Agnieszka

Tabatabaeenejad, Alireza

Tabbagh, Alain

Tadesse, Haile

Tadic, Jovan

Tagesson, Torbern

Takahashi, Kazunori

Takahashi, Masaya

Takebayashi, Hideki

Takewaka, Satoshi

Tallapragada, Vijay

Tamayo-Uria, Ibon

Tan, Jackson

Tan, Jing

Tan, Kun

Tanase, Mihai A.

Tang, Hao

Tang, Hong

Tang, Jinyun

Tang, Shijun

Tang, Wenbo

Tang, Wenqing

Tang, Youhua

Tanguy, Maliko

Tanhuanpää, Topi

Tansey, Kevin

Tansock, Joe

Tao, Lei

Tapete, Deodato

Taramelli, Andrea

Tarantino, Cristina

Tarantino, Eufemia

Taravat, Alireza

Tarnavsky, Elena

Tarolli, Paolo

Tarpanelli, Angelica

Tateishi, Ryutaro

Taubenböck, Hannes

Taylor, Jonathon

Tayyebi, Amin

Teatini, Pietro
Tebaldini, Stefano

Tegowski, Jaroslaw

Teixeira, Marcos F.S.

Teixido, Teresa

Tenenbaum, David Elliot

Teodoro, A. C.

Terra, Fabricio Da Silva

Tesei, Alessandra

Tewes, Andreas

Texeira, M.

Teza, Giordano

Thapa, Rajesh Bahadur

Thappa, Rajesh Bahadur

Thayn, Jonathan

Theeuwes, Natalie

Themistocleous, Kyriakos

Theocharis, John

Thiel, Michael

Thieler, Rob

Thomas, Katagis

Thomas, Orrin

Thomas, Sever

Thompson, David

Thompson, Elizabeth J.

Thompson, Victor

Thomson, Bradley James

Thonfeld, Frank

Thouret, Jean-Claude

Thurai, Merhala

Tian, Baijun

Tian, Bo

Tian, Feng

Tian, Jiandong

Tian, Jing

Tian, Weiming

Ticehurst, Catherine

Tien, Chung-Hao

Tilly, Nora

Timár, Gábor

Timothy, Dube

Tivy, Adrienne

Tolleson, Douglas R.

Tomljenović, Ivan 


\author{
Tompalski, Piotr \\ Tong Minh, Dinh Ho \\ Topouzelis, Konstantinos \\ Torabzadeh, Hossein \\ Torbick, Nathan \\ Toro-Farmer, Gerardo \\ Torres, Leigh \\ Torresan, Chiara \\ Torres-Sánchez, Jorge \\ Tortini, Riccardo \\ Tosi, Luigi \\ Toth, Viktor R \\ Toulios, Leonidas \\ Touzi, Ridha \\ Trabucco, Antonio \\ Tracy, Quirk \\ Tramontana, Gianluca \\ Tramutoli, Valerio \\ Tran, Chinh C. \\ Trasatti, Elisa \\ Triantakonstantis, Dimitrios \\ Trier, Øivind Due \\ Trigo, Isabel \\ Trinder, John \\ Trishchenko, Alexander \\ Trout, Thomas \\ Truckenbrodt, John \\ Truong-Hong, Linh \\ Tsagkatakis, Grigorios \\ Tsakiri-Strati, Maria \\ Tsamalis, Christoforos \\ Tschudi, Mark \\ Tseng, Kuo-Hsin \\ Tseng, Yi-Hsing \\ Tsioukas, Vassilios \\ Tsuda, Shuichi \\ Tsutsumida, Narumasa \\ $\mathrm{Tu}$, Kevin P. \\ Tucci, Grazia \\ Tuleau-Malot, Christine \\ Tullis, Jason \\ Tum, Markus \\ Tuominen, Sakari
}

Turner, Darren

Turner, David

Tyagi, Bhishma

Tyukavina, Alexandra

Tyystjärvi, Esa

Tzeng, Yu-Chang

Udelhoven, Thomas

Udo, Keiko

Ullmann, Tobias

Ulrich, Markus

Urai, Minoru

Urquhart, Erin A.

Urrego-Blanco, Jorge

Usery, Lynn

Uslander, Thomas

Utkin, Andrei

Vadrevu, Krishna

Vaglio Laurin, Gaia

Valero, Francisco

Valiente, José Antonio

Valipour, Mohammad

Vallet, Bruno

Valls, Xavier Binefa

Valova, Iren

van Aardt, Jan

van Buer, Nicholas J.

van den Hoek, Jamon

van der Kwast, J.

van der Meer, Freek

van der Meijde, Mark

van der Merwe, Deon

van der Werff, Harald

van der Zande, Dimitry

van Dervelde, Rogier

van Kessel, Thijs

van Leeuwen, Martin

van Malderen, Roeland

van Niekerk, Adriaan

van Pelt, Scott

van Wesemael, Bas

Vanderkluysen, Loyc

Vanneschi, Claudio

Vantrepotte, Vincent 
Varotsos, Costas

Vaseashta, Ashok K.

Vasilakos, Christos

Vasques, Gustavo De Mattos

Vastaranta, Mikko

Vauhkonen, Jari

Vazquez, Jorge

Vega, $M$.

Véga, Cédric

Veganzones, Miguel Angel

Velasco-Forero, Santiago

Velez-Reyes, Miguel

Veljanovski, Tatjana

Velpuri, Naga Manohar

Venafra, Sara

Vepakomma, Udayalakshmi

Vera, Ana

Verbyla, Dave

Verdonck, Lieven

Verger, Aleixandre

Verhoef, Wouter

Verhoeven, Geert

Verma, Manish

Vernier, Paolo

Veroustraete, Frank

Verrot, Lucile

Verstockt, Steven

Vescovo, Loris

Vetrivel, Anand

Vettore, Antonio

Vezzetti, Enrico

Vieira, Douglas Alexandre Gomes

Viger, Roland J.

Vignudelli, Stefano

Vila, Daniel

Vilar, Lara

Villa, Federica

Villa, Paolo

Villard, Ludovic

Villarreal, Miguel

Vincent, Robert K.

Vinukollu, Raghuveer K.

Virdis, Salvatore G. P.
Virtanen, Timo H.

Visser, Fleur

Vittek, Marian

Vittucci, Cristina

Viviani, Michele

Vlassova, Lidia

Vogelmann, James

Vogelzang, Jur

Vohland, Michael

Völkel, Jörg

Volkov, Denis L.

Volpi, Michele

Von Wehrden, Henrik

Vosselman, George

Vozel, Benoit

Vulfson, Leonid

Vuolo, Francesco

Wagle, Pradeep

Wagstaff, Kiri

Wakita, Koji

Walawender, Jakub P.

Walczykowski, Piotr

Wald, Andrew

Walker, Jessica J.

Walker, Sara Louise

Wallace, Luke

Wallentin, Gudrun

Waller, Joanne

Walsh, Kerry

Walter, Volker

Wan, Zhanming

Wang, Changcheng

Wang, Chao

Wang, Cheng

Wang, Chi-Kuei

Wang, Di

Wang, Guangxing

Wang, Guoqing

Wang, Hui

Wang, Haihong

Wang, Hongquan

Wang, Hsueh-Ching

Wang, Hua 


\begin{tabular}{|c|c|}
\hline Wang, Hui & Watson, Katelyn \\
\hline Wang, Jiao & Wdowinski, Shimon \\
\hline Wang, Juan & Wechsler, Suzanne \\
\hline Wang, Julian X. L. & Wegner, Jan \\
\hline Wang, Jun & Wei, Anhua \\
\hline Wang, Junbang & Wei, Jianwei \\
\hline Wang, Ke & Wei, Meng \\
\hline Wang, Keyan & Weigel, Katja \\
\hline Wang, Lei & Weihermüller, Lutz \\
\hline Wang, Lei & Weimin, Huang \\
\hline Wang, Likun & Weiss, Marie \\
\hline Wang, Lili & Welc, Fabian \\
\hline Wang, Lizhe & Wellens, Joost \\
\hline Wang, Lunche & Wen, Chih-Yu \\
\hline Wang, Menghua & Wen, Jianguang \\
\hline Wang, Mi & Wen, Li \\
\hline Wang, Pei & Wen, Tzai-Hung \\
\hline Wang, Ping & Wen, Yangmao \\
\hline Wang, Qi & Weng, Fuzhong \\
\hline Wang, Qunming & Wentz, Elizabeth \\
\hline Wang, Sendo & Wenzel, Helmut \\
\hline Wang, Shang & Werdell, Jeremy \\
\hline Wang, Teng & Were, Kennedy \\
\hline Wang, Weiguang & Werle, Dirk \\
\hline Wang, Weile & Werner, Piotr \\
\hline Wang, Wenhui & Westergaard-Nielsen, Andreas \\
\hline Wang, Wenlei & Wężyk, Piotr \\
\hline Wang, Xianwei & Wheate, Roger \\
\hline Wang, Xin & Whitcraft, Alyssa \\
\hline Wang, Xuhui & White, Devin \\
\hline Wang, Yao-tien & White, Lori \\
\hline Wang, Yonghui & White, Scott M. \\
\hline Wang, Yu & Whitehead, Ken \\
\hline Wang, Yujie & Whiteside, Tim \\
\hline Wang, Yunpeng & Whitley, Thomas \\
\hline Wannasiri, Wasinee & Wiegner, Matthias \\
\hline Warland, Jon S. & Wieland, Marc \\
\hline Warner, David & Wijaya, Arief \\
\hline Warren, Mark & Wilkes, Phil \\
\hline Waser, Lars & Wilkin, Paul \\
\hline Watanabe, Kenichi & Wilkinson, Ben \\
\hline Watanabe, Manabu & Wilksch, Philip \\
\hline Watkins, James & Willers, Jeffrey \\
\hline
\end{tabular}


Williams, Gabriela

Williamson, Grant

Willis, Michael John

Wills, Skye A.

Wilsey, Brian

Wimmers, Anthony

Wingate, Vladimir

Wolfe, Robert E.

Wolters, Erwin

Wong, Kwan Kit

Wouter, Verhoef

$\mathrm{Wu}$, Aisheng

$\mathrm{Wu}$, Bin

$\mathrm{Wu}, \mathrm{Bo}$

$\mathrm{Wu}$, Changshan

$\mathrm{Wu}$, Guofeng

$\mathrm{Wu}$, Hua

$\mathrm{Wu}$, Jianping

$\mathrm{Wu}$, Jianwei

$\mathrm{Wu}$, Jianyong

$\mathrm{Wu}$, Lixin

$\mathrm{Wu}$, Mingquan

$\mathrm{Wu}$, Qingbai

$\mathrm{Wu}$, Qiusheng

$\mathrm{Wu}$, Wenbin

$\mathrm{Wu}$, Wenjin

$\mathrm{Wu}$, Xiongbin

$\mathrm{Wu}$, Yonghua

$\mathrm{Wu}$, Zhisheng

Wulamu, Wasit

Wunch, Debra

Wynne, Timothy T.

Xenakis, Georgios

$X i$, Baike

Xia, Junshi

Xiao, Pengfeng

XIAO, Wen

Xie, Hongjie

Xie, Mowen

Xie, Xianhong

Xie, Zhijian

Xie, Zunyi

Xin, Qinchuan
Xu, Chong

$\mathrm{Xu}$, Dandan

$X u$, Jia

$\mathrm{Xu}$, Liang (Alan)

$\mathrm{Xu}$, Linlin

$\mathrm{Xu}$, Min

Xu, Qing

$\mathrm{Xu}$, Wenbin

$\mathrm{Xu}$, Xiaoguang

$X u, X i n$

$\mathrm{Xu}$, Xingang

$\mathrm{Xu}$, Yong

$\mathrm{Xu}$, Yongming

Xue, Xianwu

Yackel, John J.

Yagci, Ali

Yahalom, Asher

Yahya, Ghassoun

Yajima, Taro

Yamaguchi, Yasushi

Yamaguchi, Yoshio

Yamamoto, Kristina H.

Yan, Bo Kun

Yan, Dong

Yan, Lei

Yan, Lin

Yan, Wai Yeung

Yan, Yajing

Yang, Chuan-Kai

Yang, Dongxu

Yang, Guijun

Yang, Haiqing

Yang, Haoping

Yang, Hua

Yang, Jiachuan

Yang, Jinn-Min

Yang, John Xun

Yang, Kang

Yang, Song

Yang, Wen

Yang, Wenze

Yang, Xiaolei

Yang, Yuting 


\begin{tabular}{|c|c|}
\hline Yanovsky, Igor & Zema, Demetrio Antonio \\
\hline Yao, Tian & Zeng, Chuiqing \\
\hline Yao, Wei & Zha, Tianshan \\
\hline Yasuda, Takatoshi & Zhan, Wenfeng \\
\hline Yavitt, Joseph & Zhan, Yulin \\
\hline Ye, Shujun & Zhang, Bin \\
\hline Yen, Haw & Zhang, Bing \\
\hline Yen, Tian Ming & Zhang, Caiyun \\
\hline Yi, Bingqi & Zhang, Chunhua \\
\hline Yi, Shuang & Zhang, Damao \\
\hline Yılmaz, M. Tuğrul & Zhang, Geli \\
\hline Yin, $\mathrm{He}$ & Zhang, Guoqing \\
\hline Yin, Jifu & Zhang, Guosheng \\
\hline Yin, Tiangang & Zhang, Hankui \\
\hline Yin, Xiaobin & Zhang, Hao \\
\hline Yong, Bin & Zhang, Hong \\
\hline Yoon, Jongmin & Zhang, Hongsheng \\
\hline Yoshino Watanabe, Fernanda Sayuri & Zhang, Hua \\
\hline Yoshioka, Hiroki & Zhang, Huihui \\
\hline You, Xinge & Zhang, Jiahua \\
\hline You, Yalei & Zhang, Jiiashu \\
\hline Young, John & Zhang, Jingxiong \\
\hline Young, Mary & Zhang, Jixian \\
\hline Young-Gyu, Park & Zhang, Ke \\
\hline Yousef, Foad & Zhang, Kongwen \\
\hline Yousif, Osama & Zhang, Lefei \\
\hline Yu, Fangfang & Zhang, Lei \\
\hline Yu, Le & Zhang, Liangjing \\
\hline Yu, Qiang & Zhang, Liangpei \\
\hline Yu, Qin & Zhang, Lifu \\
\hline Yu, Xiaolei & Zhang, Lu \\
\hline Yu, Yifan & Zhang, Maozhen \\
\hline Yu, Yunyue & Zhang, Miao \\
\hline Yuan, Qiangqiang & Zhang, Mingyang \\
\hline Yue, Wenze & Zhang, Minwei \\
\hline Yue, Yuemin & Zhang, Qingling \\
\hline Yuichi, Hyakawa & Zhang, Qingyuan \\
\hline Yunus, Ali P. & Zhang, Shuqing \\
\hline Yurganov, Leonid & Zhang, Wuming \\
\hline Zagajewski, Bogdan & Zhang, Xi \\
\hline Zakšek, Klemen & Zhang, Xianfeng \\
\hline Zaman, Bushra & Zhang, Xiangrong \\
\hline Zatelli, Paolo & Zhang, Xiaoli \\
\hline
\end{tabular}




Zhang, Xiaotong
Zhang, Xiying
Zhang, Yan
Zhang, Yongguang
Zhang, Yongjun
Zhang, Yuanchong
Zhang, Yuanzhi
Zhao, Chaoying
Zhao, Feng
Zhao, Guangyu
Zhao, Hongrui
Zhao, Kaichun
Zhao, Lei
Zhao, Shuhe
Zhao, Tianjie
Zhao, Tianliang
Zhao, Wei
Zhao, Wenke
Zhao, Xiang
Zhen, Zhen
Zheng, Weizhong
Zhong, Liheng
Zhou, ChunXia
Zhou, Decheng

\author{
Zhou, Guoqing \\ Zhu, Junfeng \\ Zhu, Likai \\ Zhu, Lingli \\ Zhu, Qing \\ Zhu, Weining \\ Zhu, Xi \\ Zhu, Zaichun \\ Zhu, Zhe \\ Zhuo, Lu \\ Zibordi, Giuseppe \\ Zingaretti, Primo \\ Zinner, Tobias \\ Zinnert, Julie \\ Zinno, Ivana \\ Zipper, Samuel \\ Zlinszky, András \\ Zou, Xiaobo \\ Zribi, Mehrez \\ Zucca, Francesco \\ Zude, Manuela \\ Zweig, Christa L. \\ Zwieback, Simon \\ Zygielbaum, Arthur
}

